\title{
Some New Generating Functions for the Modified Laguerre Polynomials
}

\author{
Nejla Özmen* \\ Department of Mathematics, Faculty of Science and Arts, Düzce University, Düzce, \\ Turkey
}

Received 28 December 2018; Accepted (in revised version) 6 May 2019

\begin{abstract}
In this paper, we obtain some new results on bilateral generating functions of the modified Laguerre polynomials. We also get generating function relations between the modified Laguerre polynomials and the generalized Lauricella functions. Some special cases and important applications are also discussed.
\end{abstract}

AMS subject classifications: 33C45, 33C65

Key words: Modified Laguerre polynomials, generating function, multilinear and multilateral generating function, recurrence relations, generalized Lauricella function.

\section{Introduction}

In solving many problems of theoretical and mathematical physics, we often need various special functions. So, it is important to analyze a special function and to determine its characteristic properties such as its generating function relations, integral representations, explicit formula, recurrence relations, and so on. In the present paper we mainly study on the modified versions of the classical Laguerre polynomials that are famous ones in the theory of special functions. More precisely, we obtain some new results regarding bilateral generating functions of the modified Laguerre polynomials.

In the literature there are various methods of obtaining generating functions, such as group-theoretic method and analytic method (see, for instance, [3, 4,7, 18, 29,31]).

Throughout the paper we need the following definitions and notations.

The modified Laguerre polynomials, denoted by $f_{n}^{(\beta)}(x)$, are specified by the series (see [12])

$$
f_{n}^{(\beta)}(x)=\frac{(\beta)_{n}}{n !} \sum_{k=0}^{n} \frac{(-n)_{k}}{(1-n-\beta)_{k}} \frac{x^{k}}{k !},
$$

\footnotetext{
*Corresponding author.

Emails: nejlaozmen06@gmail.com, nejlaozmen@duzce.edu.tr (N. Özmen)
} 
where, as usual, $(\lambda)_{v}$ denotes the Pochhammer symbol given by (for $\lambda, v \in \mathbb{C}$ and in terms of Gamma function)

$$
(\lambda)_{v}:=\frac{\Gamma(\lambda+v)}{\Gamma(\lambda)}= \begin{cases}1, & (v=0, \lambda \in \mathbb{C} \backslash\{0\}), \\ \lambda(\lambda+1) \cdots(\lambda+n-1), & (v=n \in \mathbb{N}, \lambda \in \mathbb{C}),\end{cases}
$$

with the convention $(0)_{0}:=1$.

These polynomials have the following generating function relations (see [18]):

$$
\begin{array}{ll}
\sum_{n=0}^{\infty} f_{n}^{(\beta)}(x) t^{n}=(1-t)^{-\beta} \exp (x t), & |t|<1, \\
\sum_{n=0}^{\infty}\left(\begin{array}{c}
n+m \\
m
\end{array}\right) f_{n+m}^{(\beta)}(x) t^{n}=(1-t)^{-\beta-m} \exp (x t) f_{m}^{(\beta)}(x(1-t)), & |t|<1 .
\end{array}
$$

Another modified version of the Laguerre polynomials of degree $n$, denoted by $L_{\alpha, \beta, m, n}(x)$, was defined by Goyal [13] in the form

$$
L_{\alpha, \beta, m, n}(x)=\frac{\beta^{n}(m)_{n}}{n !} F_{1}\left(-n ; m ; \frac{\alpha x}{\beta}\right), \quad(m \neq 0,-1,-2, \cdots, \quad \beta \neq 0),
$$

where ${ }_{1} F_{1}$ is the confluent hypergeometric function (see [10]). We should note that the polynomials $L_{\alpha, \beta, m, n}(x)$ are found in an increasing number of mathematical works since it is more easier to handle and also more practical in numerical computations (see, for instance, $[17,19,26-28])$. For the polynomials in (1.3), the following generating function was obtain in [24]:

$$
\sum_{n=0}^{\infty} \frac{(k+1)_{n}}{n !} L_{\alpha, \beta, m, n+k}(x) t^{n}=(1-\beta t)^{-m-k} \exp \left(\frac{\alpha x t}{\beta t-1}\right) L_{\alpha, \beta, m, k}\left(\frac{x}{1-\beta t}\right),
$$

where $|\beta t|<1$. In recent years, many researchers have studied multilinear and multilateral generating functions for different type of polynomials, such as Altın et al. [1], Chan et al. [2], Chen et al. [5,6], Dattoil et al. [8], Erkus et al. [11] and Liu [14], Qureshi et al. [25]. Similarly, in [15] Liu et al. introduced bilateral generating functions for the Chan-ChyanSrivastava polynomials and the generalized Lauricella functions, and in [16] bilateral generating functions for the Erkus-Srivastava polynomials and generalized Lauricella functions were derived. Recently, we have obtained generating functions for the generalized Lauricella polynomials and the generalized Cesáro functions (see [23]). It is also possible to find different generating functions by means of a similar method used in [20] and [22].

With the above information, our strategy in this paper is as follows: in Section 2, we get an integral representation of the polynomials $f_{n}^{(\beta)}(x)$ and provide a new generating function relation for the polynomials $L_{\alpha, \beta, m, n}(x)$. In Section 3, we derive several families of bilinear and bilateral generating functions for the polynomials $L_{\alpha, \beta, m, n}(x)$. In Section 4 , we discuss some special cases. In Section 5, we obtain various families of bilateral generating functions for the modified Laguerre polynomials and the generalized Lauricella functions defined in [30]. The last section is devoted to the concluding remarks. 


\section{Some properties of modified Laguerre polynomials}

In this section, we first get an integral representation of the polynomials $f_{n}^{(\beta)}(x)$ defined in (1.1). Later we provide a generating function relation for the modified Laguerre polynomials $L_{\alpha, \beta, m, n}(x)$.

Theorem 2.1. The modified Laguerre polynomials $f_{n}^{(\beta)}(x)$ have the following integral representation

$$
f_{n}^{(\beta)}(x)=\frac{1}{n ! \Gamma(\beta)} \int_{0}^{\infty} e^{-u} u^{\beta-1}(u+x)^{n} d u .
$$

Proof. If we use the identity

$$
a^{-v}=\frac{1}{\Gamma(v)} \int_{0}^{\infty} e^{-a t} t^{v-1} d t(\operatorname{Re}(v)>0),
$$

on the left-hand side of the generating function relation (1.2a), then we may write that

$$
\begin{aligned}
\sum_{n=0}^{\infty} f_{n}^{(\beta)}(x) t^{n} & =\frac{1}{\Gamma(\beta)} \int_{0}^{\infty} e^{-(1-t) u} u^{\beta-1} e^{x t} d u \\
& =\frac{1}{\Gamma(\beta)} \int_{0}^{\infty} e^{-u} u^{\beta-1} \sum_{n=0}^{\infty} \frac{(u+x)^{n}}{n !} t^{n} d u \\
& =\sum_{n=0}^{\infty}\left(\frac{1}{n ! \Gamma(\beta)} \int_{0}^{\infty} e^{-u} u^{\beta-1}(u+x)^{n} d u\right) t^{n} .
\end{aligned}
$$

Comparing the coefficients of $t^{n}$, the proof is completed.

We should note that, unfortunately in the preset paper we are not able to derive a similar integral representation of the polynomials $L_{\alpha, \beta, m, n}(x)$ since it is more complicated than the process for the polynomials $f_{n}^{(\beta)}(x)$.

Now we obtain a new generating function for the modified Laguerre polynomials $L_{\alpha, \beta, m, n}(x)$.

Theorem 2.2. The polynomials $L_{\alpha, \beta, m, n}(x)$ have the following generating function relation:

$$
\sum_{n=0}^{\infty} L_{\alpha, \beta, m, n}(x) t^{n}=(1-\beta t)^{-m} \exp \left(\frac{\alpha x t}{\beta t-1}\right) .
$$

Proof. Using (1.3) on the left-hand side of (2.2), we get

$$
\begin{aligned}
\sum_{n=0}^{\infty} L_{\alpha, \beta, m, n}(x) t^{n} & =\sum_{n=0}^{\infty} \frac{\beta^{n}(m)_{n}}{n !} F_{1}\left(-n ; m ; \frac{\alpha x}{\beta}\right) t^{n} \\
& =\sum_{n=0}^{\infty} \frac{\beta^{n}(m)_{n}}{n !} \sum_{p=0}^{n} \frac{(-n)_{p}}{(m)_{p} p !}\left(\frac{\alpha x}{\beta}\right)^{p} t^{n} \\
& =(1-\beta t)^{-m} \exp \left(\frac{\alpha x t}{\beta t-1}\right),
\end{aligned}
$$


which completes the proof.

Now differentiating both sides of the relation (2.2) with respect to $x$ and also using

$$
\sum_{n=0}^{\infty} \sum_{k=0}^{\infty} A(k, n)=\sum_{n=0}^{\infty} \sum_{k=0}^{n} A(k, n-k)
$$

we get the next (differential) recurrence relation for the modified Laguerre polynomials:

$$
\beta L_{\alpha, \beta, m, n-1}^{\prime}(x)-L_{\alpha, \beta, m, n}^{\prime}(x)-\alpha L_{\alpha, \beta, m, n-1}(x)=0, \quad(n \geq 1) .
$$

It is also possible find to obtain a different recurrence relation for these polynomials by differentiating both sides of (2.2) with respect to $t$ as follows:

$$
\begin{aligned}
& \alpha x\left[\sum_{k=0}^{n} \beta^{k} L_{\alpha, \beta+(n+1) L_{\alpha, \beta, m, n+1}(x), m, n-k}(x)-\sum_{k=0}^{n-1}(k+1) \beta^{k+1} L_{\alpha, \beta, m, n-k-1}(x)\right] \\
= & m \sum_{k=0}^{n} \beta^{k+1} L_{\alpha, \beta, m, n-k}(x) .
\end{aligned}
$$

The next result is an easy consequence of Theorem 2.2, which gives a new addition formula for the modified Laguerre polynomials.

Corollary 2.1. The modified Laguerre polynomials satisfy the following addition formula

$$
L_{\alpha, \beta, m_{1}+m_{2}, n}\left(x_{1}+x_{2}\right)=\sum_{k=0}^{n} L_{\alpha, \beta, m_{1}, n-k}\left(x_{1}\right) L_{\alpha, \beta, m_{2}, k}\left(x_{2}\right) .
$$

Proof. Replacing $m$ by $m_{1}+m_{2}$ and $x$ by $x_{1}+x_{2}$ in (2.2), we observe that

$$
\begin{aligned}
& \sum_{n=0}^{\infty} L_{\alpha, \beta, m_{1}+m_{2}, n}\left(x_{1}+x_{2}\right) t^{n}=(1-\beta t)^{-m_{1}-m_{2}} \exp \left(\frac{\alpha\left(x_{1}+x_{2}\right) t}{\beta t-1}\right) \\
= & \sum_{n=0}^{\infty} L_{\alpha, \beta, m_{1}, n}\left(x_{1}\right) t^{n} \sum_{k=0}^{\infty} L_{\alpha, \beta, m_{2}, k}\left(x_{2}\right) t^{k}=\sum_{n=0}^{\infty} \sum_{k=0}^{\infty} L_{\alpha, \beta, m_{1}, n}\left(x_{1}\right) L_{\alpha, \beta, m_{2}, k}\left(x_{2}\right) t^{n+k} \\
= & \sum_{n=0}^{\infty} \sum_{k=0}^{n} L_{\alpha, \beta, m_{1}, n-k}\left(x_{1}\right) L_{\alpha, \beta, m_{2}, k}\left(x_{2}\right) t^{n} .
\end{aligned}
$$

Hence, comparing the coefficients of $t^{n}$, the proof follows, immediately.

\section{Bilinear and bilateral generating functions}

The main goal of this section is to derive several families of bilinear and bilateral generating functions for the modified Laguerre polynomials $L_{\alpha, \beta, m, n}(x)$ having the generating function relation (2.2). Note that a similar work has recently been done for other special functions, such as the Meixner polynomials and the generalized Cesàro polynomials (see $[20,23])$.

We first get the next result. 
Theorem 3.1. Corresponding to an identically non-vanishing function $\Omega_{\mu}\left(y_{1}, \cdots, y_{r}\right)$ of $r$ complex variables $y_{1}, \cdots, y_{r}(r \in \mathbb{N})$ and of complex order $\mu$, let

$$
\Lambda_{\mu, \psi}\left(y_{1}, \cdots, y_{r} ; \zeta\right):=\sum_{k=0}^{\infty} a_{k} \Omega_{\mu+\psi k}\left(y_{1}, \cdots, y_{r}\right) \zeta^{k}, \quad a_{k} \neq 0, \quad \mu, \psi \in \mathbb{C},
$$

and

$$
\Theta_{n, p}^{\mu, \psi}\left(x ; y_{1}, \cdots, y_{r} ; \xi\right):=\sum_{k=0}^{[n / p]} a_{k} L_{\alpha, \beta, m, n-p k}(x) \Omega_{\mu+\psi k}\left(y_{1}, \cdots, y_{r}\right) \xi^{k} .
$$

Then, for $p \in \mathbb{N}$, we have

$$
\sum_{n=0}^{\infty} \Theta_{n, p}^{\mu, \psi}\left(x ; y_{1}, \cdots, y_{r} ; \frac{\eta}{t^{p}}\right) t^{n}=(1-\beta t)^{-m} \exp \left(\frac{\alpha x t}{\beta t-1}\right) \Lambda_{\mu, \psi}\left(y_{1}, \cdots, y_{r} ; \eta\right) .
$$

Proof. Let $\Psi$ denote the left side of (3.1). Then,

$$
\Psi=\sum_{n=0}^{\infty} \sum_{k=0}^{[n / p]} a_{k} L_{\alpha, \beta, m, n-p k}(x) \Omega_{\mu+\psi k}\left(y_{1}, \cdots, y_{r}\right) \eta^{k} t^{n-p k} .
$$

Replacing $n$ by $n+p k$, we get

$$
\begin{aligned}
\Psi & =\sum_{n=0}^{\infty} \sum_{k=0}^{\infty} a_{k} L_{\alpha, \beta, m, n}(x) f_{n}^{\alpha}(x) \Omega_{\mu+\psi k}\left(y_{1}, \cdots, y_{r}\right) \eta^{k} t^{n} \\
& =\sum_{n=0}^{\infty} L_{\alpha, \beta, m, n}(x) t^{n} \sum_{k=0}^{\infty} a_{k} \Omega_{\mu+\psi k}\left(y_{1}, \cdots, y_{r}\right) \eta^{k} \\
& =(1-\beta t)^{-m} \exp \left(\frac{\alpha x t}{\beta t-1}\right) \Lambda_{\mu, \psi}\left(y_{1}, \cdots, y_{r} ; \eta\right),
\end{aligned}
$$

which is the desired result.

We also get the following theorem.

Theorem 3.2. Corresponding to an identically non-vanishing function $\Omega_{\mu}\left(y_{1}, \cdots, y_{r}\right)$ of $r$ complex variables $y_{1}, \cdots, y_{r}(r \in \mathbb{N})$ and of complex order $\mu$, let

$$
\Lambda_{\mu, \psi}^{n, p}\left(x_{1}+x_{2} ; y_{1}, \cdots, y_{r} ; z\right):=\sum_{k=0}^{[n / p]} a_{k} L_{\alpha, \beta, m_{1}+m_{2}, n-p k}\left(x_{1}+x_{2}\right) \Omega_{\mu+\psi k}\left(y_{1}, \cdots, y_{r}\right) z^{k},
$$

where $a_{k} \neq 0, \mu, \psi \in \mathbb{C}, n, p \in \mathbb{N}$. Then, we have

$$
\begin{aligned}
& \sum_{k=0}^{n} \sum_{l=0}^{[k / p]} a_{l} L_{\alpha, \beta, m_{1}, n-k}\left(x_{1}\right) L_{\alpha, \beta, m_{2}, k-p l}\left(x_{2}\right) \Omega_{\mu+\psi l}\left(y_{1}, \cdots, y_{r}\right) z^{l} \\
= & \Lambda_{\mu, \psi}^{n, p}\left(x_{1}+x_{2} ; y_{1}, \cdots, y_{r} ; z\right) .
\end{aligned}
$$


Proof. Let $\omega$ denote the left side of the assertion (3.2). Then, it follows from (2.3) that

$$
\begin{aligned}
\mathcal{W} & =\sum_{l=0}^{[n / p] n-p l} \sum_{k=0}^{n} a_{l} L_{\alpha, \beta, m_{1}, n-k-p l}\left(x_{1}\right) L_{\alpha, \beta, m_{2}, k}\left(x_{2}\right) \Omega_{\mu+\psi l}\left(y_{1}, \cdots, y_{r}\right) z^{l} \\
& =\sum_{l=0}^{[n / p]} a_{l}\left(\sum_{k=0}^{n-p l} L_{\alpha, \beta, m_{1}, n-k-p l}\left(x_{1}\right) L_{\alpha, \beta, m_{2}, k}\left(x_{2}\right)\right) \Omega_{\mu+\psi l}\left(y_{1}, \cdots, y_{r}\right) z^{l} \\
& =\sum_{l=0}^{[n / p]} a_{l} L_{\alpha, \beta, m_{1}+m_{2}, n-p l}\left(x_{1}+x_{2}\right) \Omega_{\mu+\psi l}\left(y_{1}, \cdots, y_{r}\right) z^{l} \\
& =\Lambda_{\mu, \psi}^{n, p}\left(x_{1}+x_{2} ; y_{1}, \cdots, y_{r} ; z\right) .
\end{aligned}
$$

Hence, the proof is completed.

In a similar manner, we get the next result.

Theorem 3.3. Corresponding to an identically non-vanishing function $\Omega_{\mu}\left(y_{1}, \cdots, y_{r}\right)$ of $r$ complex variables $y_{1}, \cdots, y_{r}(r \in \mathbb{N})$ and of complex order $\mu$, let

$$
\Lambda_{\mu, q, \psi}\left(x ; y_{1}, \cdots, y_{r} ; t\right):=\sum_{k=0}^{\infty} a_{k} L_{\alpha, \beta, m, p+q k}(x) \Omega_{\mu+\psi k}\left(y_{1}, \cdots, y_{r}\right) t^{k},
$$

where $a_{n} \neq 0, \mu \in \mathbb{C}$, and

$$
\theta_{n, q}^{\mu, \psi}\left(y_{1}, \cdots, y_{r} ; z\right):=\sum_{k=0}^{[n / q]} \frac{(k+1)_{n-q k}}{(n-q k) !} a_{k} \Omega_{\mu+\psi k}\left(y_{1}, \cdots, y_{r}\right) z^{k} .
$$

Then, for $p \in \mathbb{N}$, we have

$$
\begin{aligned}
& \sum_{n=0}^{\infty} L_{\alpha, \beta, m, n+p}(x) \theta_{n, p, q}\left(y_{1}, \cdots, y_{r} ; z\right) t^{n} \\
= & (1-\beta t)^{-m-p} \exp \left(\frac{\alpha x t}{\beta t-1}\right) \Lambda_{m \mu}^{p, q}\left(\frac{x}{1-\beta t} ; y_{1}, \cdots, y_{r} ; z\left(\frac{t}{1-\beta t}\right)^{q}\right) .
\end{aligned}
$$

Proof. Let $\kappa$ denote the left side of (3.3). Then, we may write that

$$
\kappa=\sum_{n=0}^{\infty} L_{\alpha, \beta, m, n+p}(x) \sum_{k=0}^{[n / q]} \frac{(k+1)_{n-q k}}{(n-q k) !} a_{k} \Omega_{\mu+\psi k}\left(y_{1}, \cdots, y_{r}\right) z^{k} t^{n} .
$$

Replacing $n$ by $n+q k$ and then using (1.2b),

$$
\begin{aligned}
\kappa & =\sum_{n=0}^{\infty} \sum_{k=0}^{\infty} \frac{(k+1)_{n}}{n !} L_{\alpha, \beta, m, n+p+q k}(x) a_{k} \Omega_{\mu+\psi k}\left(y_{1}, \cdots, y_{r}\right) z^{k} t^{n+q k} \\
& =\sum_{k=0}^{\infty}\left(\sum_{n=0}^{\infty} \frac{(k+1)_{n}}{n !} L_{\alpha, \beta, m, n+p+q k}(x) t^{n}\right) a_{k} \Omega_{\mu+\psi k}\left(y_{1}, \cdots, y_{r}\right)\left(z t^{q}\right)^{k}
\end{aligned}
$$




$$
\begin{aligned}
& =\sum_{k=0}^{\infty}(1-\beta t)^{-m-p-q k} \exp \left(\frac{\alpha x t}{\beta t-1}\right) \\
& \quad \times L_{\alpha, \beta, m, p+q k}\left(\frac{x}{1-\beta t}\right) a_{k} \Omega_{\mu+\psi k}\left(y_{1}, \cdots, y_{r}\right)\left(z t^{q}\right)^{k} \\
& =(1-\beta t)^{-m-p} \exp \left(\frac{\alpha x t}{\beta t-1}\right) \\
& \quad \times \sum_{k=0}^{\infty} a_{k} L_{\alpha, \beta, m, k+q k}\left(\frac{x}{1-\beta t}\right) \Omega_{\mu+\psi k}\left(y_{1}, \cdots, y_{r}\right)\left(\frac{z t^{q}}{(1-\beta t)^{q}}\right)^{k} \\
& =(1-\beta t)^{-m-p} \exp \left(\frac{\alpha x t}{\beta t-1}\right) \Lambda_{m \mu}^{p, q}\left(\frac{x}{1-\beta t^{\prime}} ; y_{1}, \cdots, y_{r} ; z\left(\frac{t}{1-\beta t}\right)^{q}\right),
\end{aligned}
$$

which completes the proof.

\section{Special cases}

If the multivariable function $\Omega_{\mu+\psi k}\left(y_{1}, \cdots, y_{r}\right), k \in \mathbb{N}_{0}, r \in \mathbb{N}$, is expressed in terms of simpler functions of one and more variables, then we can give further applications of the above theorems. For example, taking

$$
\Omega_{\mu+\psi k}\left(y_{1}, \cdots, y_{r}\right)=\Phi_{\mu+\psi k}^{(\alpha)}\left(y_{1}, \cdots, y_{r}\right)
$$

in Theorem 3.1, where the multivariable polynomials $\Phi_{\mu+\psi k}^{(\alpha)}\left(x_{1}, \cdots, x_{r}\right)$ are generated by (see [21])

$$
\left(1-x_{1} t\right)^{-\alpha} e^{\left(x_{2}+\cdots+x_{r}\right) t}=\sum_{n=0}^{\infty} \Phi_{n}^{(\alpha)}\left(x_{1}, \cdots, x_{r}\right) t^{n}, \quad\left(\alpha \in \mathbb{C} ; \quad|t|<\left\{\left|x_{1}\right|^{-1}\right\}\right) .
$$

we obtain the following result, which provides a bilateral generating function for multivariate polynomials $\Phi_{\mu+\psi k}^{(\alpha)}\left(x_{1}, \cdots, x_{r}\right)$ and the modified Laguerre polynomials.

Corollary 4.1. If

$$
\Lambda_{\mu, \psi}\left(y_{1}, \cdots, y_{r} ; \zeta\right):=\sum_{k=0}^{\infty} a_{k} \Phi_{\mu+\psi k}^{(\alpha)}\left(y_{1}, \cdots, y_{r}\right) \zeta^{k}, \quad\left(a_{k} \neq 0, \quad \mu, \psi \in \mathbb{C}\right),
$$

then,

$$
\begin{aligned}
& \sum_{n=0}^{\infty} \sum_{k=0}^{[n / p]} a_{k} L_{\alpha, \beta, m, n-p k}(x) \Phi_{\mu+\psi k}^{(v)}\left(y_{1}, \cdots, y_{r}\right) \frac{\eta^{k}}{t^{p k}} t^{n} \\
= & (1-\beta t)^{-m} \exp \left(\frac{\alpha x t}{\beta t-1}\right) \Lambda_{\mu, \psi}\left(y_{1}, \cdots, y_{r} ; \eta\right) .
\end{aligned}
$$


Remark 4.1. Using the generating relation (4.1) for the multivariable polynomials $\Phi_{\mu+\psi k}^{(\alpha)}\left(x_{1}, \cdots, x_{r}\right)$ and putting $a_{k}=1, \mu=0, \psi=1$ in Corollary 4.1 , we observe that

$$
\begin{aligned}
& \sum_{n=0}^{\infty} \sum_{k=0}^{[n / p]} L_{\alpha, \beta, m, n-p k}(x) \Phi_{k}^{(v)}\left(y_{1}, \cdots, y_{r}\right) \eta^{k} t^{n-p k} \\
= & (1-\beta t)^{-m} \exp \left(\frac{\alpha x t}{\beta t-1}\right)\left(1-y_{1} \eta\right)^{-v} e^{\left(y_{2}+\cdots+y_{r}\right) \eta,}
\end{aligned}
$$

where $|\beta t|<1, v \in \mathbb{C},|\eta|<\left\{\left|y_{1}\right|^{-1}\right\}$.

If we set

$$
r=1 \quad \text { and } \quad \Omega_{\mu+\psi k}\left(y_{1}\right)=L_{\alpha, \beta, m_{3}, \mu+\psi k}\left(x_{3}\right)
$$

in Theorem 3.2, then we have the following bilinear generating functions for the modified Laguerre polynomials.

Corollary 4.2. If

$$
\begin{aligned}
& \Lambda_{\mu, \psi}^{n, p}\left(x_{1}+x_{2} ; x_{3} ; z\right) \\
= & : \sum_{k=0}^{[n / p]} a_{k} L_{\alpha, \beta, m_{1}+m_{2}, n}\left(x_{1}+x_{2}\right) f_{n-p k}^{\left(\alpha_{1}+\alpha_{2}\right)}\left(x_{1}+x_{2}\right) L_{\alpha, \beta, m_{3}, \mu+\psi k}\left(x_{3}\right) z^{k},
\end{aligned}
$$

where $a_{k} \neq 0, \mu, \psi \in \mathbb{C}$, then

$$
\sum_{k=0}^{n} \sum_{l=0}^{[k / p]} a_{l} L_{\alpha, \beta, m_{1}, n}\left(x_{1}\right) L_{\alpha, \beta, m_{2}, n}\left(x_{2}\right) L_{\alpha, \beta, m_{3}, \mu+\psi k}\left(x_{3}\right) z^{l}=\Lambda_{\mu, \psi}^{n, p}\left(x_{1}+x_{2} ; x_{3} ; z\right) .
$$

Remark 4.2. Using (2.3) and also taking $a_{l}=z=1, \mu=0, \psi=1, p=1$ in Corollary 4.2, we get

$$
\sum_{k=0 l=0}^{n} \sum_{\alpha, \beta, m_{1}, n-k}^{k}\left(x_{1}\right) L_{\alpha, \beta, m_{2}, k-l}\left(x_{2}\right) L_{\alpha, \beta, m_{3}, l}\left(x_{3}\right)=L_{\alpha, \beta, m_{1}+m_{2}+m_{3}, n}\left(x_{1}+x_{2}+x_{3}\right) .
$$

If we set

$$
r=1 \quad \text { and } \quad \Omega_{\mu+\psi k}(y)=P_{\mu+\psi k}^{(\alpha, \beta)}(y)
$$

in Theorem 3.3 and taking into account the classical Jacobi polynomials generated by (see [10])

$$
\sum_{n=0}^{\infty} P_{n}^{(\alpha, \beta)}(x) t^{n}=\frac{2^{\alpha+\beta}}{\rho}(1-t+\rho)^{-\alpha}(1+t+\rho)^{-\beta},
$$

where $\rho=\left(1-2 x t+t^{2}\right)^{1 / 2}$, then we get a family of the bilateral generating functions for the classical Jacobi polynomials and the modified Laguerre polynomials as follows. 
Corollary 4.3. If

$$
\begin{aligned}
& \Lambda_{m, q}(x ; y ; t)=: \sum_{k=0}^{\infty} a_{k} L_{\alpha, \beta, m, p+q k}(x) P_{\mu+\psi k}^{(\alpha, \beta)}(y) t^{k}, \quad\left(a_{k} \neq 0, \quad m \in \mathbb{N}_{0}, \quad k \neq 0\right), \\
& \theta_{n, m, q}^{\mu, \psi}(y ; z):=\sum_{k=0}^{[n / q]} \frac{(k+1)_{n-q k}}{(n-q k) !} a_{k} P_{\mu+\psi k}^{(\alpha, \beta)}(y) z^{k},
\end{aligned}
$$

where $n, p \in \mathbb{N}$, then we have

$$
\begin{aligned}
& \sum_{n=0}^{\infty} L_{\alpha, \beta, m, n+p}(x) \theta_{n, m, q}^{\mu, \psi}(y ; z) t^{n} \\
= & (1-\beta t)^{-m-p} \exp \left(\frac{\alpha x t}{\beta t-1}\right) \Lambda_{m, q}\left(\frac{x}{1-\beta t} ; y ; z\left(\frac{t}{1-\beta t}\right)^{q}\right) .
\end{aligned}
$$

Furthermore, for every suitable choice of the coefficients $a_{k}\left(k \in \mathbb{N}_{0}\right)$ if the multivariable functions $\Omega_{\mu+\psi k}\left(y_{1}, \cdots, y_{r}\right), r \in \mathbb{N}$, are expressed as an appropriate product of several simpler functions, the assertions of Theorems 3.1, 3.2 and 3.3 can be applied in order to derive various families of multilinear and multilateral generating functions for the modified Laguerre polynomials.

\section{Some new bilateral generating functions for modified Laguerre polynomials}

In this section, we derive various families of bilateral generating functions for the modified Laguerre polynomials and the generalized Lauricella functions defined in [30].

Recall that a generalization of the familiar Kampé de Fériet hypergeometric function in two variables, which is called the generalized Lauricella function (or, the Srivastava and Daoust function), was introduced by Srivastava and Daoust as follows (see [30]):

$$
\begin{aligned}
& F_{C: D^{(1)} ; \cdots ; D^{(n)}}^{A: B^{(1)}, \cdots ; B^{(n)}}\left(\begin{array}{rl}
{\left[(a): \theta^{(1)}, \cdots, \theta^{(n)}\right]:\left[\left(b^{(1)}\right): \phi^{(1)}\right] ; \cdots ;} & {\left[\left(b^{(n)}\right): \phi^{(n)}\right] ;} \\
{\left[(c): \psi^{(1)}, \cdots, \psi^{(n)}\right]:\left[\left(d^{(1)}\right): \delta^{(1)}\right] ; \cdots ;} & {\left[\left(d^{(n)}\right): \delta^{(n)}\right] ;}
\end{array}\right) \\
= & \sum_{m_{1}, \cdots, m_{n}=0}^{\infty} \Omega\left(m_{1}, \cdots, m_{n}\right) \frac{z_{1}^{m_{1}}}{m_{1} !} \cdots \frac{z_{n}^{m_{n}}}{m_{n} !},
\end{aligned}
$$

where

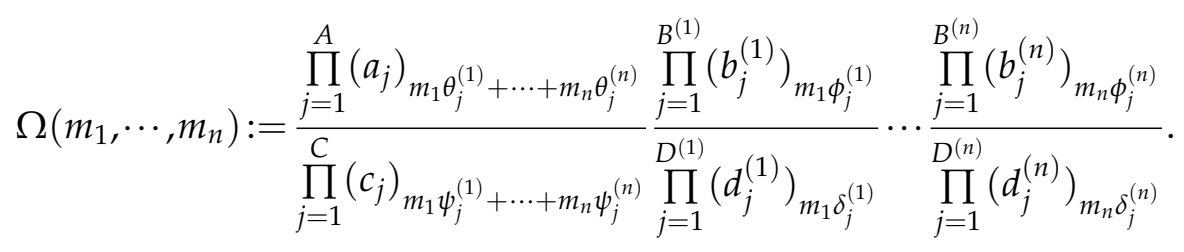


Here, the coefficients

$$
\begin{aligned}
& \theta_{j}^{(k)}(j=1, \cdots, A ; k=1, \cdots, n) \quad \text { and } \quad \phi_{j}^{(k)}\left(j=1, \cdots, B^{(k)} ; k=1, \cdots, n\right), \\
& \psi_{j}^{(k)}(j=1, \cdots, C ; k=1, \cdots, n) \quad \text { and } \delta_{j}^{(k)}\left(j=1, \cdots, D^{(k)} ; k=1, \cdots, n\right),
\end{aligned}
$$

are real constants and $\left(b_{B^{(k)}}^{(k)}\right)$ abbreviates the array of $B^{(k)}$ parameters

$$
b_{j}^{(k)}, \quad\left(j=1, \cdots, B^{(k)} ; k=1, \cdots, n\right),
$$

with similar interpretations for other sets of parameters [16].

For a suitable bounded non-vanishing multiple sequence $\left\{\Omega\left(m_{1}, \cdots, m_{s}\right)\right\}_{m_{1}, \cdots, m_{s} \in \mathbb{N}_{0}}$ of real or complex parameters, let $\phi_{n}\left(u_{1} ; u_{2}, \cdots, u_{s}\right)$ be a function of $s$ real or complex variables defined by

$$
\begin{aligned}
\phi_{n}\left(u_{1} ; u_{2}, \cdots, u_{s}\right)=: \sum_{m_{1}=0}^{n} & \sum_{m_{2}, \cdots, m_{s}=0}^{\infty} \frac{(-n)_{m_{1}}\left(m_{1}+1\right)_{n-m_{1}}((b))_{m_{1} \phi}}{((d))_{m_{1} \delta}} \\
& \times \Omega\left(f\left(m_{1}, \cdots, m_{s}\right), m_{2}, \cdots, m_{s}\right) \frac{u_{1}^{m_{1}}}{m_{1} !} \cdots \frac{u_{s}^{m_{s}}}{m_{s} !},
\end{aligned}
$$

where, for convenience,

$$
((b))_{m_{1} \phi}=\prod_{j=1}^{B}\left(b_{j}\right)_{m_{1} \phi_{j}} \quad \text { and } \quad((d))_{m_{1} \delta}=\prod_{j=1}^{D}\left(d_{j}\right)_{m_{1} \delta_{j}} .
$$

Then, we get the next result.

Theorem 5.1. The following generating function relation holds true:

$$
\begin{aligned}
& \sum_{n=0}^{\infty} L_{\alpha, \beta, m, n}(x) \phi_{n}\left(u_{1} ; u_{2}, \cdots, u_{s}\right) \frac{t^{n}}{n !} \\
= & (1-\beta t)^{-m} \exp \left(\frac{\alpha x t}{\beta t-1}\right) \sum_{m_{1}, p, m_{2}, \cdots, m_{s}=0}^{\infty} \frac{(m)_{m_{1}+p}((b))_{\left(m_{1}+p\right) \phi}}{(1)_{m_{1}+p}((d))_{\left(m_{1}+p\right) \delta}(m)_{p}} \\
& \quad \times \Omega\left(f\left(m_{1}, \cdots, m_{s}\right), m_{2}, \cdots, m_{s}\right) \frac{\left(\frac{-u_{1} \beta t}{1-\beta t}\right)^{m_{1}}}{m_{1} !} \frac{\left(\frac{u_{1} \alpha x t}{(1-\beta t)^{2}}\right)^{p}}{p !} \frac{u_{2}^{m_{2}}}{m_{2} !} \cdots \frac{u_{s}^{m_{s}}}{m_{s} !},
\end{aligned}
$$

where $\phi_{n}\left(u_{1} ; u_{2}, \cdots, u_{s}\right)$ is given by (5.1).

Proof. By using (1.2b), it is easy to see that

$$
\begin{aligned}
& \sum_{n=0}^{\infty} L_{\alpha, \beta, m, n}(x) \phi_{n}\left(u_{1} ; u_{2}, \cdots, u_{s}\right) \frac{t^{n}}{n !} \\
= & \sum_{n=0}^{\infty} L_{\alpha, \beta, m, n}(x) \sum_{m_{1}=0 m_{2}, \cdots, m_{s}=0}^{n} \sum^{\infty} \frac{(-n)_{m_{1}}\left(m_{1}+1\right)_{n-m_{1}}((b))_{m_{1} \phi}}{((d))_{m_{1} \delta}} \\
& \times \Omega\left(f\left(m_{1}, \cdots, m_{s}\right), m_{2}, \cdots, m_{s}\right) \frac{u_{1}^{m_{1}}}{m_{1} !} \cdots \frac{u_{s}^{m_{s}}}{m_{s} !} \frac{t^{n}}{n !}
\end{aligned}
$$




$$
\begin{aligned}
& =\sum_{m_{1}, m_{2}, \cdots, m_{s}=0}^{\infty}(1-\beta t)^{-m-m_{1}} \exp \left(\frac{\alpha x t}{\beta t-1}\right) L_{\alpha, \beta, m, m_{1}}\left(\frac{x}{1-\beta t}\right) \\
& \quad \times \frac{((b))_{m_{1} \phi}}{((d))_{m_{1} \delta}} \Omega\left(f\left(m_{1}, \cdots, m_{s}\right), m_{2}, \cdots, m_{s}\right) \frac{\left(-u_{1} t\right)^{m_{1}}}{m_{1} !} \frac{u_{2}^{m_{2}}}{m_{2} !} \cdots \frac{u_{s}^{m_{s}}}{m_{s} !} \\
& =(1-\beta t)^{-m} \exp \left(\frac{\alpha x t}{\beta t-1}\right) \sum_{m_{1}, p, m_{2}, \cdots, m_{s}=0}^{\infty} \frac{(m)_{m_{1}+p}((b))_{\left(m_{1}+p\right) \phi}}{(1)_{m_{1}+p}((d))_{\left(m_{1}+p\right) \delta}(m)_{p}} \\
& \quad \times \Omega\left(f\left(m_{1}+p, \cdots, m_{s}\right), m_{2}, \cdots, m_{s}\right) \frac{\left(\frac{-u_{1} \beta t}{1-\beta t}\right)^{m_{1}}}{m_{1} !} \frac{\left(\frac{u_{1} \alpha x t}{(1-\beta t)^{2}}\right)^{p}}{p !} \frac{u_{2}^{m_{2}}}{m_{2} !} \cdots \frac{u_{s}^{m_{s}}}{m_{s} !},
\end{aligned}
$$

which completes the proof.

By appropriately choosing the multiple sequence $\Omega$ in Theorem 5.1, we can obtain some interesting results which give bilateral generation functions for modified Laguerre polynomials $L_{\alpha, \beta, m, n}(x)$ and the generalized Lauricella functions.

Upon setting

$$
\Omega\left(f\left(m_{1}, \cdots, m_{s}\right), m_{2}, \cdots, m_{s}\right)=\frac{(a)_{m_{1}+\cdots+m_{s}}\left(b_{2}\right)_{m_{2}} \cdots\left(b_{s}\right)_{m_{s}}}{(m)_{m_{1}} \cdots\left(c_{s}\right)_{m_{s}}}
$$

and

$$
\phi=\delta=0 \quad\left(\text { that is, } \phi_{1}=\cdots=\phi_{B}=\delta_{1}=\cdots=\delta_{D}=0\right),
$$

in Theorem 5.1, we get the following result.

Corollary 5.1. The following bilateral generating function is satisfied:

$$
\begin{aligned}
& \sum_{n=0}^{\infty} L_{\alpha, \beta, m, n}(x) F_{A}^{(s)}\left[a,-n, b_{2}, \cdots, b_{s} ; m, \cdots, c_{s} ; u_{1}, \cdots, u_{s}\right] \frac{t^{n}}{n !} \\
& =(1-\beta t)^{-m} \exp \left(\frac{\alpha x t}{\beta t-1}\right) F_{1: 0 ; 1 ; 1 ; \cdots ; 1}^{1: 0 ; 1 ; \cdots ; 1}\left(\begin{array}{ccc}
{[(a): 1, \cdots, 1]:} & -; & -; \\
{\left[(1): \varphi^{(1)}, \cdots, \varphi^{(s+1)}\right]:} & -; & {[m: 1] ;}
\end{array}\right. \\
& \begin{array}{l}
\left.\left[b_{2}: 1\right] ; \cdots ; \quad\left[b_{s}: 1\right] ; \quad\left(\frac{-u_{1} \beta t}{1-\beta t}\right),\left(\frac{u_{1} \alpha x t}{(1-\beta t)^{2}}\right), u_{2}, \cdots, u_{s}\right), \\
{\left[c_{2}: 1\right] ; \cdots ; \quad\left[c_{s}: 1\right] ;}
\end{array}
\end{aligned}
$$

where $F_{A}^{(s)}$ is the Lauricella function and the coefficients $\varphi^{(\omega)}$ are

$$
\varphi^{(\omega)}= \begin{cases}1, & (1 \leq \omega \leq 2) \\ 0, & (2<\omega \leq s+1) .\end{cases}
$$

Now, it is also possible to obtain bilateral generating functions for the modified Laguerre polynomials $f_{n}^{(\beta)}(x)$. To see this, for a suitable bounded non-vanishing multiple sequence

$$
\left\{\Omega\left(m_{1}, m_{2}, \cdots, m_{s}\right)\right\}_{m_{1}, m_{2}, \cdots, m_{s} \in \mathbb{N}_{0}}
$$


of real or complex parameters, let $\Xi_{n}\left(u_{1} ; u_{2}, \cdots, u_{s}\right)$ be a function of $s$ real or complex variables $u_{1} ; u_{2}, \cdots, u_{s}$ defined by

$$
\begin{aligned}
& \Xi_{n}\left(u_{1} ; u_{2}, \cdots, u_{s}\right) \\
= & : \sum_{m_{1}=0 m_{2}, \cdots, m_{s}=0}^{n} \sum^{\infty} \frac{(-n)_{m_{1}}((b))_{m_{1} \phi}}{((d))_{m_{1} \delta}} \Omega\left(f\left(m_{1}, \cdots, m_{s}\right), m_{2}, \cdots, m_{s}\right) \frac{u_{1}^{m_{1}}}{m_{1} !} \cdots \frac{u_{s}^{m_{s}}}{m_{s} !},
\end{aligned}
$$

where $((b))_{m_{1} \phi}$ and $((d))_{m_{1} \delta}$ are as stated before.

The we obtain the next result.

Theorem 5.2. The following bilateral generating function holds:

$$
\begin{aligned}
& \sum_{n=0}^{\infty} f_{n}^{(\beta)}(x) \Xi_{n}\left(u_{1} ; u_{2}, \cdots, u_{s}\right) t^{n} \\
= & (1-t)^{-\beta} \exp (x t) \sum_{m_{1}, p, m_{2}, \cdots, m_{s}=0}^{\infty} \frac{((b))_{\left(m_{1}+p\right) \phi}(\beta)_{m_{1}}}{((d))_{\left(m_{1}+p\right) \delta}} \\
& \quad \times \Omega\left(f\left(\left(m_{1}+p\right), \cdots, m_{s}\right), m_{2}, \cdots, m_{s}\right) \frac{\left(\frac{u_{1} t}{t-1}\right)^{m_{1}}}{m_{1} !} \frac{\left(-u_{1} x t\right)^{p}}{p !} \frac{u_{2}^{m_{2}}}{m_{2} !} \cdots \frac{u_{s}^{m_{s}}}{m_{s} !},
\end{aligned}
$$

where $\Xi_{n}\left(u_{1} ; u_{2}, \cdots, u_{s}\right)$ is given by (5.2).

Proof. By using (1.2b), we observe that

$$
\begin{aligned}
& \sum_{n=0}^{\infty} f_{n}^{(\beta)}(x) \Xi_{n}\left(u_{1} ; u_{2}, \cdots, u_{s}\right) t^{n} \\
= & \sum_{n=0}^{\infty} f_{n}^{(\beta)}(x) \sum_{m_{1}=0}^{n} \sum_{m_{2}, \cdots, m_{s}=0}^{\infty} \frac{(-n)_{m_{1}}((b))_{m_{1} \phi}}{((d))_{m_{1}} \delta} \\
& \times \Omega\left(f\left(m_{1}, \cdots, m_{s}\right), m_{2}, \cdots, m_{s}\right) \frac{u_{1}^{m_{1}}}{m_{1} !} \cdots \frac{u_{s}^{m_{s}}}{m_{s} !} t^{n} \\
= & \sum_{m_{1}, m_{2}, \cdots, m_{s}=0}^{\infty} \frac{((b))_{m_{1} \phi}}{((d))_{m_{1} \delta}} \Omega\left(f\left(m_{1}, \cdots, m_{s}\right), m_{2}, \cdots, m_{s}\right) \\
& \times\left(-u_{1} t\right)^{m_{1}} \frac{u_{2}^{m_{2}}}{m_{2} !} \cdots \frac{u_{s}^{m_{s}}}{m_{s} !}(1-t)^{-\beta-m_{1}} \exp (x t) f_{m_{1}}^{(\beta)}(x(1-t)),
\end{aligned}
$$

which gives

$$
\begin{aligned}
& \sum_{n=0}^{\infty} f_{n}^{(\beta)}(x) \Xi_{n}\left(u_{1} ; u_{2}, \cdots, u_{s}\right) t^{n} \\
= & (1-t)^{-\beta} \exp (x t) \sum_{m_{1}, m_{2}, \cdots, m_{s}=0}^{\infty} \frac{((b))_{m_{1} \phi}}{((d))_{m_{1} \delta}} \Omega\left(f\left(m_{1}, \cdots, m_{s}\right), m_{2}, \cdots, m_{s}\right) \\
& \left.\times\left(-u_{1} t\right)^{m_{1}} \frac{u_{2}^{m_{2}}}{m_{2} !} \cdots \frac{u_{s}^{m_{s}}}{m_{s} !}(1-t)^{-m_{1}} \sum_{p=0}^{m_{1}} \frac{(\beta)_{m_{1}-p}}{\left(m_{1}-p\right) ! p !}(x(1-t))\right)^{p}
\end{aligned}
$$




$$
\begin{aligned}
& =(1-t)^{-\beta} \exp (x t) \sum_{m_{1}, p, m_{2}, \cdots, m_{s}=0}^{\infty} \frac{((b))_{\left(m_{1}+p\right) \phi}(\beta)_{m_{1}}}{((d))_{\left(m_{1}+p\right) \delta}} \\
& \quad \times \Omega\left(f\left(\left(m_{1}+p\right), \cdots, m_{s}\right), m_{2}, \cdots, m_{s}\right) \frac{\left(\frac{u_{1} t}{t-1}\right)^{m_{1}}}{m_{1} !} \frac{\left(-u_{1} x t\right)^{p}}{p !} \frac{u_{2}^{m_{2}}}{m_{2} !} \cdots \frac{u_{s}^{m_{s}}}{m_{s} !} .
\end{aligned}
$$

Hence, the proof is completed.

By appropriately choosing the multiple sequence $\Omega$ in Theorem 5.2 , it is possible to get some bilateral generation function relations between modified Laguerre polynomials $f_{n}^{(\beta)}(x)$ and the generalized Lauricella functions. We should that such results have recently been obtained for other special polynomials in $[1,7,11,14-17,20-23,26,29]$.

I. Letting

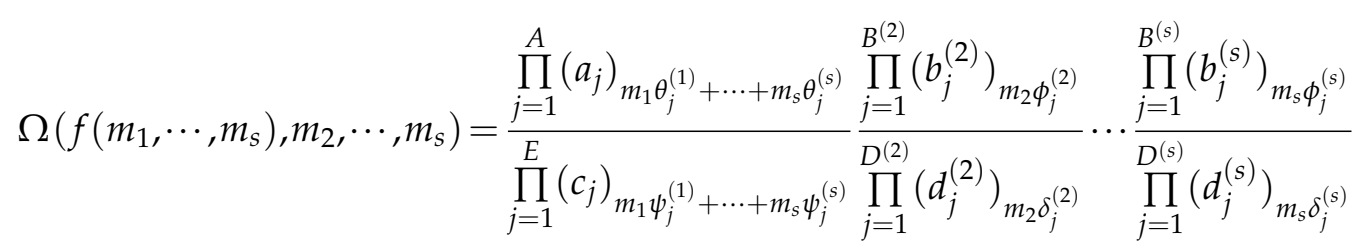

in Theorem 5.2, we obtain the next result.

Corollary 5.2. The following bilateral generating function is satisfied:

$$
\begin{aligned}
& \sum_{n=0}^{\infty} f_{n}^{(\beta)}(x) F_{E: D ; D^{(2)} ; \cdots ; D^{(s)}}^{A: B+1 B^{(2)} ; \ldots ;(s)}
\end{aligned}
$$

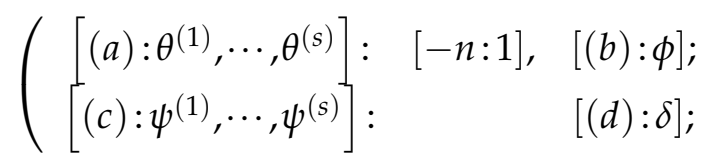

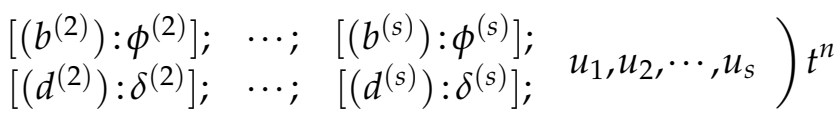

$$
\begin{aligned}
& =(1-t)^{-\beta} \exp (x t) F_{E+D: 0 ; 0 ; D^{(2)} ; \cdots ; D^{(s)}}^{A+B: 1 ; 0 ; B^{(2)} ; \ldots ; B^{(s)}} \\
& \left(\begin{array}{cccc}
{\left[(e): \varphi^{(1)}, \cdots, \varphi^{(s+1)}\right]:} & {[\beta: 1],} & -; & {\left[\left(b^{(2)}\right): \phi^{(2)}\right] ;} \\
{\left[(f): \xi^{(1)}, \cdots, \xi^{(s+1)}\right]:} & - & -; & {\left[\left(d^{(2)}\right): \delta^{(2)}\right] ;}
\end{array}\right. \\
& \left.\begin{array}{ll}
\cdots ; & {\left[\left(b^{(s)}\right): \phi^{(s)}\right] ;} \\
\cdots ; & {\left[\left(d^{(s)}\right): \delta^{(s)}\right] ;}
\end{array} \quad\left(\frac{u_{1} t}{t-1}\right),\left(-u_{1} x t\right), u_{2}, \cdots, u_{s}\right),
\end{aligned}
$$

where the coefficients $e_{j}, f_{j}, \varphi_{j}^{(k)}$ and $\xi_{j}^{(k)}$ are respectively given by

$$
e_{j}=\left\{\begin{array}{ll}
a_{j}, & (1 \leq j \leq A), \\
b_{j-A}, & (A<j \leq A+B),
\end{array} \quad f_{j}= \begin{cases}c_{j}, & (1 \leq j \leq E), \\
d_{j-E}, & (E<j \leq E+D),\end{cases}\right.
$$




$$
\varphi_{j}^{(r)}=\left\{\begin{array}{ll}
\theta_{j}^{(1)}, & (1 \leq j \leq A ; 1 \leq r \leq 2), \\
\theta_{j}^{(r-1)}, & (1 \leq j \leq A ; 2<r \leq s+1), \\
\phi_{j-A}, & (A<j \leq A+B ; 1 \leq r \leq 2), \\
0, & (A<j \leq A+B ; 2<r \leq s+1),
\end{array} \quad \xi_{j}^{(r)}= \begin{cases}\psi_{j}^{(1)}, & (1 \leq j \leq E ; 1 \leq r \leq 2), \\
\psi_{j}^{(r-1)}, & (1 \leq j \leq E ; 2<r \leq s+1), \\
\delta_{j-E}, & (E<j \leq E+D ; 1 \leq r \leq 2) \\
0, & (E<j \leq E+D ; 2<r \leq s+1) .\end{cases}\right.
$$

II. Upon setting

$$
\begin{aligned}
& \Omega\left(f\left(m_{1}, \cdots, m_{s}\right), m_{2}, \cdots, m_{s}\right)=\frac{(a)_{m_{1}+\cdots+m_{s}}\left(b_{2}\right)_{m_{2}} \cdots\left(b_{s}\right)_{m_{s}}}{\left(c_{1}\right)_{m_{1}} \cdots\left(c_{s}\right)_{m_{s}}}, \\
& \left.\phi=\delta=0 \quad \text { (that is, } \phi_{1}=\cdots=\phi_{B}=\delta_{1}=\cdots=\delta_{D}=0\right),
\end{aligned}
$$

in Theorem 5.2, we obtain the following result.

Corollary 5.3. We have

$$
\begin{aligned}
& \sum_{n=0}^{\infty} f_{n}^{(\beta)}(x) F_{A}^{(s)}\left[a,-n, b_{2}, \cdots, b_{s} ; c_{1}, \cdots, c_{s} ; u_{1}, \cdots, u_{s}\right] t^{n} \\
& =(1-t)^{-\beta} \exp (x t) F_{1: 0 ; 0 ; 1 ; \cdots ; 1}^{1: 1 ; 0 ; 1 ; \cdots ; 1} \\
& \left(\begin{array}{cccc}
{[(a): 1, \cdots, 1]:} & {[\beta: 1] ;} & -; & {\left[b_{2}: 1\right] ;} \\
{\left[\left(c_{1}\right): \psi^{(1)}, \cdots, \psi^{(s+1)}\right]:} & -; & -; & {\left[c_{2}: 1\right] ;}
\end{array}\right. \\
& \left.\begin{array}{ll}
\cdots ; & {\left[b_{s}: 1\right] ;} \\
\cdots ; & {\left[c_{s}: 1\right] ;}
\end{array}\left(\frac{u_{1} t}{t-1}\right),\left(-u_{1} x t\right), u_{2}, \cdots, u_{s}\right),
\end{aligned}
$$

where $F_{A}^{(s)}$ is the Lauricella function and the coefficients $\psi^{(\eta)}$ are

$$
\psi^{(\eta)}= \begin{cases}1, & (1 \leq \eta \leq 2) \\ 0, & (2<\eta \leq s+1) .\end{cases}
$$

III. Taking

$$
\begin{aligned}
& \Omega\left(f\left(m_{1}, \cdots, m_{s}\right), m_{2}, \cdots, m_{s}\right) \\
& =\frac{\left(a_{1}^{(1)}\right)_{m_{2}} \cdots\left(a_{1}^{(s-1)}\right)_{m_{s}}\left(a_{2}^{(1)}\right) m_{2} \cdots\left(a_{2}^{(s-1)}\right) m_{s}}{(c) m_{1}+\cdots+m_{s}}, \\
& B=1, \quad b_{1}=b, \quad \phi_{1}=1 \quad \text { and } \quad \delta=0,
\end{aligned}
$$

in Theorem 5.2, we immediately get the following.

Corollary 5.4. The following bilateral generating function is satisfied:

$$
\sum_{n=0}^{\infty} f_{n}^{(\beta)}(x) F_{B}^{(s)}\left[-n, a_{1}^{(1)}, \cdots, a_{1}^{(s-1)}, b, a_{2}^{(1)}, \cdots, a_{2}^{(s-1)} ; c ; u_{1}, \cdots, u_{s}\right] t^{n}
$$




$$
\begin{aligned}
& =(1-t)^{-\beta} \exp (x t) F_{1: 0 ; 0 ; 0 ; \cdots ; 0}^{1: 1 ; 0 ; 2 ; 2} \\
& \left(\begin{array}{cccc}
{\left[(b): \theta^{(1)}, \cdots, \theta^{(s+1)}\right]:} & {[\beta: 1] ;} & -; & {\left[a^{(1)}: 1\right]} \\
{[(c): 1, \cdots, 1]:} & -; & -; & -;
\end{array}\right. \\
& \left.\begin{array}{cc}
\ldots ; & {\left[a^{(s-1)}: 1\right] ; \quad\left(\frac{u_{1} t}{t-1}\right),\left(-u_{1} x t\right), u_{2}, \cdots, u_{s}} \\
\ldots ; & -;
\end{array}\right),
\end{aligned}
$$

where $F_{B}^{(s)}$ is the Lauricella function and the coefficients $\theta^{(\eta)}$ are

$$
\theta^{(\eta)}= \begin{cases}1, & (1 \leq \eta \leq 2) \\ 0, & (2<\eta \leq s+1) .\end{cases}
$$

IV. Finally, letting

$$
\begin{aligned}
& \Omega\left(f\left(m_{1}, \cdots, m_{s}\right), m_{2}, \cdots, m_{s}\right)=\frac{(a)_{m_{1}+\cdots+m_{s}}\left(b_{2}\right)_{m_{2}} \cdots\left(b_{s}\right)_{m_{s}}}{(c)_{m_{1}+\cdots+m_{s}}}, \\
& \phi=\delta=0,
\end{aligned}
$$

in Theorem 5.2, we have the next result.

Corollary 5.5. The following bilateral generating function equality holds:

$$
\begin{aligned}
& \sum_{n=0}^{\infty} f_{n}^{(\beta)}(x) F_{D}^{(s)}\left[a,-n, b_{2}, \cdots, b_{s} ; c ; u_{1}, \cdots, u_{s}\right] t^{n} \\
= & (1-t)^{-\beta} \exp (x t) \\
& \quad \times F_{D}^{(s+1)}\left[a, \beta,-, b_{2}, \cdots, b_{s} ; c ;\left(\frac{u_{1} t}{t-1}\right),\left(-u_{1} x t\right), u_{2}, \cdots, u_{s}\right],
\end{aligned}
$$

where $F_{D}^{(s)}$ is a Lauricella function of s-variable.

\section{Conclusions}

In this paper, the modified Laguerre polynomials and their generating function relations are investigated. Especially, some families of multilinear and multilateral generating functions and their miscellaneous properties are obtained. We also discuss some applications and special cases. Using our approach in this paper, we see that it is possible to obtain bilinear and bilateral generating functions between the modified Laguerre polynomials and other special polynomials.

\section{Acknowledgements}

The author would especially like to thank Prof. Esra Erkus-Duman for fruitful discussions. The author would also thank the reviewer for providing insightful comments and reading the manuscript carefully. 


\section{References}

[1] A. Altin AND E. ERKUS, On a multivariable extension of the Lagrange-Hermite polynomials, Integral Transforms Spec. Funct., 17 (2006), pp. 239-244.

[2] W.-CH. C. ChAn, CH.-J. ChyAn AND H. M. SRIVASTAVA, The Lagrange polynomials in several variables, Integral Transforms Spec. Funct., 12 (2001), pp. 139-148.

[3] S. K. CHATTERJEA, Unification of a class of bilateral generating relations for certain special functions, Bull. Cal. Math. Soc., 67 (1975), pp. 115-127.

[4] S. K. CHATTERJEA, An extension of a class of bilateral generating functions for certain special functions, Bull. Inst. Math. Acad. Sinica, 5(2) (1977), pp. 323-331.

[5] K.-Y. CHEN, S.-J. LIU AND H. M. SRIVASTAVA, Some new results for the Lagrange polynomials in several variables, ANZIAM J., 49 (2007), pp. 243-258.

[6] K.-Y. CHEN AND H. M. SRIVASTAVA, A new result for hypergeometric polynomials, Proc. Amer. Math. Soc., 133 (2005), pp. 3295-3302.

[7] A. K. CHONGDAR, On the unification of a class of bilateral generating functions for certain special functions, Tamkang J. Math., 18(3) (1987), pp. 53-59.

[8] G. DAtTOIL, P. E. RiCCI AND C. CESARANO, The Lagrange polynomials, the associated generalizations, and the umbral calculus, Integral Transforms Spec. Funct., 14 (2003), pp. 181-186.

[9] A. ERdélyi, W. Magnus, F. Oberhettinger And F. G. Tricomi, Higher Transcendental Functions, Vol. I, McGraw-Hill, New York, Toronto and London, 1953.

[10] A. ERdélyi, W. Magnus, F. Oberhettinger And F. G. Tricomi, Higher Transcendental Functions, Vol. III, McGraw-Hill Book Company, New York, Toronto, London, 1955.

[11] E. ERKUS AND H. M. SRIVASTAVA, A unified presentation of some families of multivariable polynomials, Integral Transforms Spec. Funct., 17 (2006), pp. 267--273.

[12] C. C. FENG, Some generating functions of modified Laguerre polynomials, Hokkaido Math. J., 7 (1978), pp. 189-197.

[13] G. K. GOYAL, Modified Laguerre polynomials, Vijnana Parishad Anusandhan Patrica, 26 (1983), pp. 263-266.

[14] S.-J. LIU, Bilateral generating functions for the Lagrange polynomials and the Lauricella functions, Integral Transforms Spec. Funct., 20 (2009), pp. 519-527.

[15] S.-J. LIU, C.-J. CHYAN, H.-C. LU AND H. M. SRIVASTAVA, Bilateral generating functions for the Chan-Chyan-Srivastava polynomials and the generalized Lauricella functions, Integral Transforms Spec. Funct., 23(7) (2012), pp. 539-549.

[16] S.-J. LIU, S.-D. LIN, H. M. SRIVASTAVA AND M.-M. WONG, Bilateral generating functions for the Erkus-Srivastava polynomials and the generalized Lauricella functions, Appl. Math. Comput., 218 (2012), pp. 7685-7693.

[17] A. B. MAJUMDAR, On generating functions of modified Laguerre polynomials, Bull. Cal. Math. Soc., 90 (1998), pp. 163-166.

[18] E. B. MCBRIDE, Obtaining Generating Functions, Springer Tracts in Natural Philosophy, Vol. 21, Springer-Verlag, Berlin-Heidelberg-New York, 1971.

[19] W. Miller, Lie Theory and Special Functions, Academic Press, New York and London, 1986.

[20] N. ÖZMEN, Some new properties of the Meixner polynomials, Sakarya University Journal of Science, 21(6) (2017), pp. 1454-1462.

[21] N. ÖZMEN AND E. ERKUS-DUMAN, Some results for a family of multivariable polynomials, AIP Conference Proceedings, 1558 (2013), pp. 1124-1127.

[22] N. ÖZMEN AND E. ERKUS-DUMAN, On the Poisson-Charlier polynomials, Serdica Math. J., 41 
(2015), pp. 457-470.

[23] N. ÖZMEN AND E. ERKUS-DUMAN, Some families of generating functions for the generalized Cesáro polynomials, J. Comput. Anal. Appl., 25(4) (2018), pp. 670-683.

[24] M. A. PATHAN AND S. KHAN, Special linear group and modified Laguerre functions, Kyungpook Math. J., 40 (2000), pp. 1-8.

[25] M. I. QURESHI, M. S. KHAN AND M. A. PATHAN, Some multiple Gaussian hypergeometric generalizations of Buschman-Srivastava theorem, Int. J. Math. Math. Sci., 2005 (2005), pp. 143153.

[26] B. K. SEN AND A. K. CHONGDAR, On bilateral generating function of modified Laguerre polynomials from the Lie group view point, Rev. Acad. Canar. Ciencias, 5 (1993), pp. 149-156.

[27] R. SHARMA, Some generating functions of modified Laguerre polynomials-A Lie algebraic approach, Bull. Cal. Math. Soc., 82 (1990), pp. 126-130.

[28] S. N. SINGH AND R. N. BALA, Group-theoretic origins of certain generating functions of the modified Laguerre polynomials, Indian J. Pure Appl. Math., 17 (1986), pp. 512-521.

[29] J. P. Singhal AND H. M. SRIVASTAVA, A class of bilateral generating functions for certain classical polynomials, Pacific J. Math., 42 (1972), pp. 355-362.

[30] H. M. SRIVASTAVA AND M. C. DAOUST, Certain generalized Neumann expansions associated with the Kampé de Fériet function, Nederl. akad. Westensch. Indag. Math., 31 (1969), pp. 449457.

[31] L. WEISNER, Group theoretic origin of certain generating functions, Pacific J. Math., 5 (1955), pp. 1033-1039. 